\title{
Deexcitation Model for Sputtered Excited Neutral Atoms
}

\author{
B. I. Craig \\ J. P. Baxter \\ J. Singh \\ G A. Schick \\ P. H. Kobrin
}

See next page for additional authors

Follow this and additional works at: https://bearworks.missouristate.edu/articles-cnas

\section{Recommended Citation}

Craig, B. I., J. P. Baxter, J. Singh, G. A. Schick, P. H. Kobrin, Barbara Jane Garrison, and Nicholas Winograd. "Deexcitation model for sputtered excited neutral atoms." Physical review letters 57, no. 11 (1986).

This article or document was made available through BearWorks, the institutional repository of Missouri State University. The work contained in it may be protected by copyright and require permission of the copyright holder for reuse or redistribution.

For more information, please contact BearWorks@library.missouristate.edu. 
Authors

B. I. Craig, J. P. Baxter, J. Singh, G A. Schick, P. H. Kobrin, B. J. Garrison, and N. Winograd 


\title{
Deexcitation Model for Sputtered Excited Neutral Atoms
}

\author{
B. I. Craig, ${ }^{(a)}$ J. P. Baxter, J. Singh, G. A. Schick, ${ }^{(b)}$ P. H. Kobrin, ${ }^{(c)}$ B. J. Garrison, and N. Winograd \\ The Pennsylvania State University, Department of Chemistry, University Park, Pennsylvania 16802 \\ (Received 6 June 1986)
}

\begin{abstract}
Velocity distributions of In atoms in their ground $\left({ }^{2} P_{1 / 2}\right)$ and excited $\left({ }^{2} P_{3 / 2}\right)$ states ejected from In foils by bombardment with $5-\mathrm{keV} \mathrm{Ar}^{+}$ions are measured with a multiphoton resonanceionization technique. We show for the first time that the ${ }^{2} P_{3 / 2}$ level is not significantly populated during sputtering and that the only path for population of this state is via laser-induced photodissociation of $\mathrm{In}_{2}$ during the detection process. A new deexcitation model for sputtered excited neutral atoms is proposed which is based on the electronic localization of the fine-structure orbital.
\end{abstract}

PACS numbers: $79.20 . \mathrm{Nc}, 32.80 . \mathrm{Rm}, 33.80 . \mathrm{Gj}, 36.40 .+\mathrm{d}$

The measurement of velocity distributions of excited atoms desorbed from ion-bombarded solids has been of long-standing interest since these distributions hold the key to unraveling the physical mechanisms which lead to the excitation. Early studies suggested that the average kinetic energy of excited atoms was many hundreds of electronvolts larger than the ground state and was characterized by a kinetic-energy threshold. $^{1}$ Later, more sophisticated laser Doppler-shifted fluorescence techniques ${ }^{2}$ showed that the velocity distribution of the fine-structure excited levels of transition elements such as $\mathrm{Zr}^{3}$ and $\mathrm{Fe}^{4}$ with excitation energies between 0.1 and $1.0 \mathrm{eV}$ are similar to the ground-state distributions. On the other hand, $\mathrm{Ba}{ }^{1} D$ and ${ }^{3} D,{ }^{5} \mathrm{Ca}{ }^{3} P_{2},{ }^{2}$ and $\mathrm{Fe}^{5} F_{5}{ }^{6}$ metastable excited levels were found to exhibit velocity distributions whose peak intensities occur at significantly larger values than those of the corresponding ground states. In none of these later studies was there found to be evidence for a kinetic energy threshold. The shift in the excitedatom velocity distribution relative to the ground state has been commonly ascribed to an exponential velocity-dependent nonradiative deexcitation. ${ }^{4}$ The total population of these excited fine-structure levels has been explained by use of a Boltzmann distribution with an effective temperature of order $1000 \mathrm{~K}$. With this scarcity of data, it has not been possible to formulate a general theory of neutral-atom excitation following energetic ion bombardment.

In this Letter, we report velocity distributions of indium atoms ejected from polycrystalline In foil after bombardment with 5-keV $\mathrm{Ar}^{+}$ions. By means of a special multiphoton resonance-ionization (MPRI) technique ${ }^{7}$ these distributions were determined for the In ${ }^{2} P_{1 / 2}$ ground state, the ${ }^{2} P_{3 / 2}$ metastable finestructure excited state which lies $0.3 \mathrm{eV}$ above the ${ }^{2} P_{1 / 2}$ ground state, and the $\operatorname{In}_{2}{ }^{1} \Sigma_{\mathrm{g}}^{+}$ground state. The results show that the ${ }^{2} P_{3 / 2}$ level is not significantly populated during sputtering, and that the only path for the population of this state is via laser-induced photodissociation of $\operatorname{In}_{2}$. These results demonstrate, for the first time, that excitation probabilities are strongly dependent on electronic structure. Further, the observations from In, together with those from $\mathrm{Zr}, \mathrm{Fe}, \mathrm{Ca}$, and $\mathrm{Ba}$, allow us to propose a new qualitative model for nonradiative deexcitation of the desorbing atom which is based upon the degree of electronic localization of the relevant fine-structure orbital.

The experimental MPRI system and the procedure for obtaining velocity distributions have been described in detail. ${ }^{8}$ Briefly, a 200 -ns pulse of $2.5 \times 10^{6}$ ions is focused to a $2-\mathrm{mm}$ spot on the sample. Upon impact of the ion pulse, an ion extraction field is activated to remove spurious charged particles. A 6-ns laser pulse with power of $\sim 1 \mathrm{~mJ}$ and a shape of $1 \times 6$ $\mathrm{mm}$ is positioned $1.5 \mathrm{~cm}$ above and parallel to the target. This pulse ionizes a small volume of neutral particles at a time $\tau_{E}$ after the ion-pulse impact, thus defining their time of flight. The ionized particles are then accelerated by the extraction field and arrive at the front of a microchannel plate assembly at a time $\tau_{M}$ which is governed by the mass-to-charge ratio of the ion. After digital processing of the phosphor screen image and by variation of $\tau_{E}$ it is possible to determine the velocity distribution, angular distribution, and mass of the ejected neutral species.

The spectral MPRI response from sputtered In metal consists of a combination of signals arising from ground- and excited-state $\mathrm{In}$ atoms as well as from $\mathrm{In}_{2}$ molecules. ${ }^{7}$ The relevant electronic states for these species and several two-photon resonant atomic ionization schemes of interest are shown schematically in Fig. 1. For example, the 325.9-nm scheme corresponds to resonant excitation of an In atom in the ${ }^{2} P_{3 / 2}$ state to the ${ }^{2} D_{3 / 2}$ state with a photon wavelength of $325.9 \mathrm{~nm}$, followed by the subsequent (nonresonant) absorption of a second photon to form the ion, $\mathrm{In}^{+}$. We will refer to the ionization schemes by the resonant-excitation wavelength $\lambda_{e}$ value, i.e., $410.3,304.0,325.9$, and $325.6 \mathrm{~nm}$. Note that for all four schemes, light of wavelength $\lambda_{e}$ is sufficient to ionize the atom from the intermediate level although 


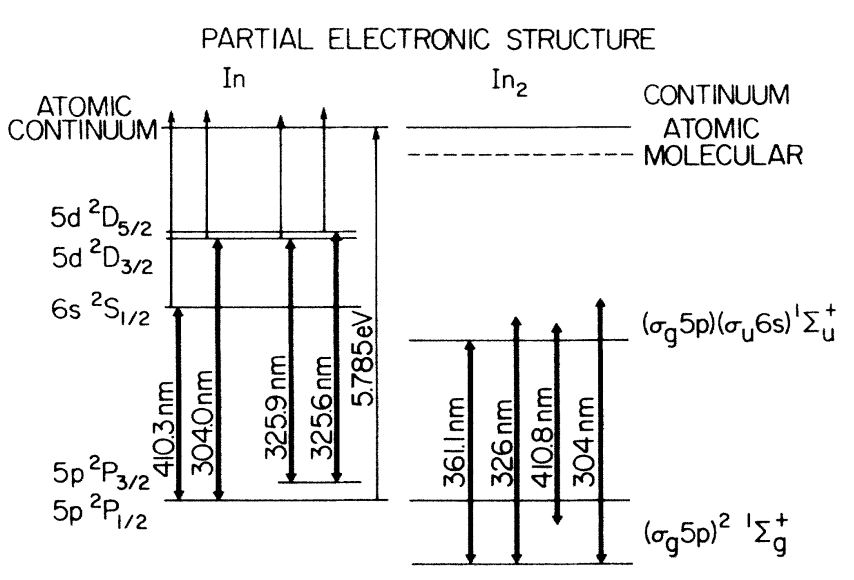

FIG. 1. Schematic diagram of the relevant energy levels of atomic In and molecular $\operatorname{In}_{2}$.

usually longer-wavelength light is used. ${ }^{7}$ Light of wavelength $\lambda_{e}$ in all schemes is also sufficient to ionize atoms which may exist in the $\left(6 s^{2} S_{1 / 2}\right)$ state.

The known (and relevant) portions of the $\mathrm{In}_{2}$ electronic spectrum are shown in Fig. 1. The ground ${ }^{1} \Sigma_{\mathrm{g}}^{+}$ state correlates to ground-state In atoms and is bound by $\sim 1 \mathrm{eV} .{ }^{9}$ One observed excited state, ${ }^{1} \Sigma_{\mathrm{u}}^{+}$, is 361.1 $\mathrm{nm}$ above the ground state. ${ }^{10}$ This state dissociates to one In atom in the $\left(6 s^{2} S_{1 / 2}\right)$ excited state and one In atom in one of the ${ }^{2} P_{J}$ states where $J=\frac{1}{2}$ or $\frac{3}{2}$. A set of ${ }^{1} \Pi_{u},{ }^{3} \Pi_{u}$, and ${ }^{3} \Sigma_{u}^{+}$states may also form from these same atomic states. These molecular states should be energetically near the ${ }^{1} \Sigma_{\mathrm{u}}^{+}$state, but their exact positions are not known. As a result of the violent nature of the sputtering process, $\mathrm{In}_{2}$ molecules in the ground electronic state manifold will be in a number of vibrational and rotational levels. Consequently, a span of wavelengths can resonantly excite $\operatorname{In}_{2} \rightarrow \operatorname{In}_{2}{ }^{*}$. From experimental observations there is resonance absorption in the 410- through 326-nm regions and not in the 304-nm wavelength range. ${ }^{7,10}$ As shown in Fig. 1, the absorption at $410 \mathrm{~nm}$ is presumably from an excited rovibrational state. Finally, as a result of the binding energy of $\mathrm{In}_{2}{ }^{+}$there should be a molecular continuum slightly below the atomic continuum.

With this understanding of the $\mathrm{In}_{2}$ electronic spectrum, the possible interactions of the laser light with the molecule can be analyzed. For example, as seen from Fig. 1, the absorption of one photon excites the molecule as

$$
\left.\operatorname{In}_{2}\left[\sigma_{\mathrm{g}} 5 p\right)^{2}{ }^{1} \Sigma_{\mathrm{g}}^{+}\right] \stackrel{\lambda_{e}}{\rightarrow} \operatorname{In}_{2}{ }^{*}\left[\left(\sigma_{\mathrm{g}} 5 p\right)\left(\sigma_{\mathrm{u}} 6 s\right)^{1} \Sigma_{\mathrm{u}}^{+}\right],
$$

where the ${ }^{1} \Sigma_{\mathrm{u}}^{+}$level may be either a bound or a dissociating state. The bound state may absorb a second photon of wavelength $\lambda_{i}$ to become ionized to $\mathrm{In}_{2}{ }^{+}$. The dissociating state, on the other hand, will yield a

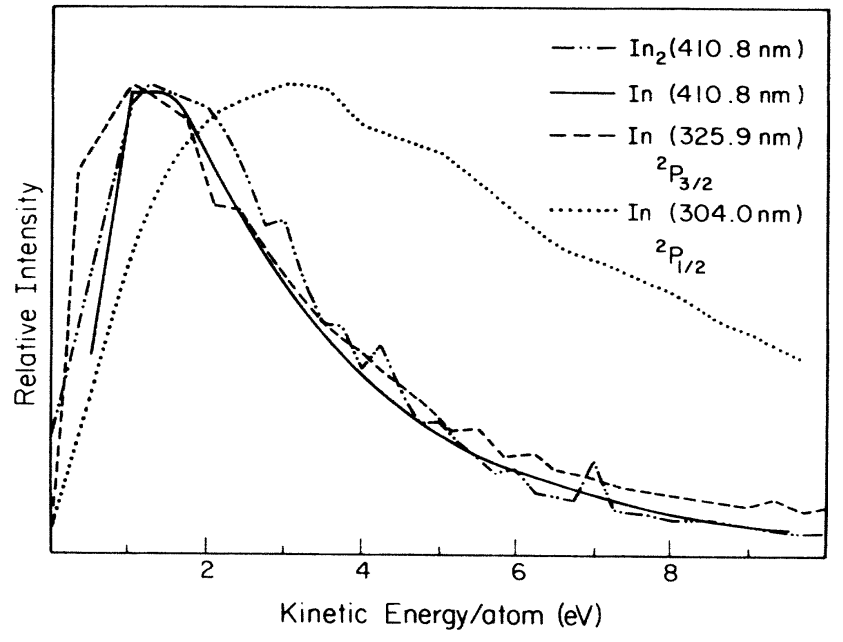

FIG. 2. Energy distributions of various In species. The yields are peak normalized to unity and are displayed as functions of KE/atom. From Ref. 13 the energy-integrated intensities are approximately as follows: 1 for $\mathrm{In}^{+}$off resonance at $410.8 \mathrm{~nm} ; 6$ for $\mathrm{In}^{+}$on resonance at $410.3 \mathrm{~nm} ; 1.2$ for $\mathrm{In}_{2}{ }^{+}$at $410.8 \mathrm{~nm} ; 200$ for $\mathrm{In}^{+}$at $304.0 \mathrm{~nm}$; and 20 for $\mathrm{In}^{+}\left({ }^{2} P_{3 / 2}\right)$ at $325.9 \mathrm{~nm}$. Dotted line: $\mathrm{In}^{+}$ionized from ground-state $\operatorname{In}\left({ }^{2} P_{1 / 2}\right)$ via the $304.0-\mathrm{nm}$ scheme. Dashdotted line: $\mathrm{In}_{2}{ }^{+}$ionized from $\mathrm{In}_{2}$ via $410.8-\mathrm{nm}$ light. Solid line: In from dissociation of ${\operatorname{~} n_{2}}^{*}$ which was excited by 410.8- $\mathrm{nm}$ light. Dashed line: $\mathrm{In}^{+}$ionized from excited-state In $\left({ }^{3} P_{3 / 2}\right)$ via the $325.9-\mathrm{nm}$ scheme.

pair of In atoms,

$$
\begin{aligned}
\operatorname{In}_{2}{ }^{*}\left[\left(\sigma_{\mathrm{g}} 5 p\right)\left(\sigma_{\mathrm{u}} 6 s\right)^{1} \Sigma_{\mathrm{u}}^{+}\right] & \\
& \rightarrow \operatorname{In}\left({ }^{2} P_{J}\right)+\operatorname{In}\left({ }^{2} S_{1 / 2}\right),
\end{aligned}
$$

or the excited dimer $\operatorname{In}_{2}{ }^{*}$ may predissociate into the above pair of In atoms through the crossing of the ${ }^{1} \Sigma_{u}^{+}$ potential energy curve with one of the three nearby states. Following the predissociation or dissociation process, a second photon may ionize the ${ }^{2} S_{1 / 2}$ atomic state yielding a distribution of $\mathrm{In}^{+}$ions which have originated from dissociated $\mathrm{In}_{2}$ molecules. From the above discussion, then, it is clear that the population of the various atomic states are linked via photochemically induced $\operatorname{In}_{2}$ dissociation processes.

We begin with an analysis of the kinetic energy (KE) distribution of ground-state $\operatorname{In}\left({ }^{2} P_{1 / 2}\right)$ atoms using the $304.0-\mathrm{nm}$ ionization scheme. This scheme is particularly straightforward since there is apparently no important contribution from dimer ionization or dissociation at this wavelength. ${ }^{7}$ The results, as shown in Fig. 2, are typical for distributions expected as a consequence of momentum transfer following the ionimpact event ${ }^{11}$ and have been thoroughly analyzed. ${ }^{12}$

The characteristics of the dimer photodissociation may be examined around the $\lambda_{e}=406$ to $414 \mathrm{~nm}$ 
range where $\mathrm{In}_{2}$ has been shown to exhibit strong absorption. ${ }^{7,10,13}$ In this spectral region MPRI of $\operatorname{In}\left({ }^{2} P_{1 / 2}\right)$ atoms occurs resonantly to the ${ }^{2} S_{1 / 2}$ state at $410.3 \mathrm{~nm}$. The resulting $\mathrm{In}^{+}$ion signal in this wavelength region, however, exhibits a spectral shape similar to the $\mathrm{In}_{2}{ }^{+}$distribution, i.e., quite broad. ${ }^{13}$ This observation suggests that the signal slightly away from $410.3 \mathrm{~nm}$ arises exclusively from ionization and dissociation of the $\operatorname{In}_{2}$ precursor molecules in accord with Eq. (2), followed by ionization of $\operatorname{In}\left({ }^{2} S_{1 / 2}\right)$.

It is possible to confirm this point by analysis of the KE distribution of $\mathrm{In}_{2}$ and In obtained slightly off the ${ }^{2} P_{1 / 2} \hookrightarrow{ }^{2} S_{1 / 2}(410.3 \mathrm{~nm})$ resonance. The species $\mathrm{In}_{2}$ and In are expected to have quite different KE distributions since $\mathrm{In}_{2}$ is believed to form by a recombination of independently ejected In atoms in the near surface region. ${ }^{7,14}$ As a result of this recombination, the dimer KE distribution is expected to generally consist of lower-KE particles and will be missing the high-KE tail characteristic of monomer distributions. The 410$\mathrm{nm}$ region is most appropriate for this test since the energy at this wavelength is not sufficient to ionize resonantly In atoms which may exist in either ${ }^{2} P_{3 / 2}$ or ${ }^{2} P_{1 / 2}$. As shown in Fig. 2, the apparent In and $\operatorname{In}_{2}$ distributions (labeled $410.8 \mathrm{~nm}$ ) are essentially identical with a shape more characteristic of molecularly rather than atomically ejected species. These results clearly show, then, that the apparent $\mathrm{In}^{+}$ion signal observed off resonance in the $410-\mathrm{nm}$ spectral range arises exclusively from $\operatorname{In}\left({ }^{2} S_{1 / 2}\right)$ produced by photodissociation of $\mathrm{In}_{2}$ as given in Eq. (2).

The most intriguing situation arises from analysis of the MPRI response from the low-lying excited-state fine-structure level, $\operatorname{In}\left({ }^{2} P_{3 / 2}\right)$, when ionized via either ${ }^{2} D_{J}$ multiplet in the 326-nm range. For these types of states, as noted earlier, it is expected that the KE distribution should peak at some higher $\mathrm{KE}$ value than the ground-state species because of an exponential velocity-dependent nonradiative deexcitation. As seen in Fig. 2, however, the KE distribution for $\operatorname{In}\left({ }^{2} P_{3 / 2}\right)$ when ionized at $\lambda_{e}=325.9 \mathrm{~nm}$ actually peaks at lower $\mathrm{KE}$ values than $\operatorname{In}\left({ }^{2} P_{1 / 2}\right)$. There does not appear to be a second peak in the $\mathrm{In}^{+}\left({ }^{2} P_{3 / 2}, 325.9 \mathrm{~nm}\right)$ curve in Fig. 2 at higher energies than the peak of the groundstate curve.

We have shown that the $\mathrm{In}_{2}$ dimers are photodissociated and that at least the $\operatorname{In}\left({ }^{2} S_{1 / 2}\right)$ dissociation product has a KE distribution almost identical to the dimer distribution. We have also shown that the $\operatorname{In}\left({ }^{2} P_{3 / 2}\right) \mathrm{KE}$ distribution is the same as the dimer distribution and quite different from the ground-state ${ }^{2} P_{1 / 2}$ distribution. As stated earlier, it is not expected that dimers and monomers will have the same KE distribution. The apparent ${ }^{2} P_{3 / 2}$ distribution, then, must be almost entirely derived from the dimer dissociation process, rather than from sputtered atoms in the ${ }^{2} P_{3 / 2}$ levels.
It is of interest to examine the physical nature of the photodissociation process and the abundances of the ${ }^{2} P_{1 / 2}$ and ${ }^{2} P_{3 / 2}$ products. This relative fraction depends on the photoabsorption cross sections as well as on the correlation of the molecular dimer levels with the atomic components. To determine this correlation consideration of nonadiabatic effects involving the potential energy curves of several molecular configurations is required. This type of dissociation process has been examined for alkali dimers where careful studies of this correlation have been undertaken. ${ }^{15,16}$ Our experiments are not particularly suited to this type of analysis since the dimer species are ejected from the surface in a range of rotational and vibrational levels. It will be of future interest, however, to see if these levels can be directly measured and related to the physical nature of the sputtering process itself.

The factors that are important in determining whether an atom in an excited state will relax as it departs from the surface are becoming apparent. In one limit are fine-structure states such as the ${ }^{5} D_{J}$, $3 d^{6} 4 s^{2}$ manifold of $\mathrm{Fe}$ and the ${ }^{3} F_{J}, 4 d^{2} 5 s^{2}$ manifold of $\mathrm{Zr}$. The fine-structure states are determined by $d$ electron coupling and not by s-electron coupling. In these cases the $s$ orbitals are larger than the $d$ orbitals, and effectively shield the $d$ electrons from interaction with the metal conduction band as the atom departs from the surface. Consequently, relaxation processes have long lifetimes and the velocity distribution is independent of atomic state.

In the intermediate regime are the ${ }^{5} F, 3 d^{7} 4 s^{1}$ state of $\mathrm{Fe}$, the ${ }^{3} P_{2}, 4 s^{1} 4 p^{1}$ state of $\mathrm{Ca}$, and the ${ }^{1,3} \mathrm{D}, 6 s^{1} 5 d^{1}$ states of $\mathrm{Ba}$. Here the velocity distributions of the excited states are broader than the ground-state distribution. Of note is that the $\mathrm{Ba}$ distributions depend on the electronic environment of the original matrix ${ }^{5}$ i.e., $\mathrm{Ba}$ metal, $\mathrm{BaO}$, or $\mathrm{BaF}_{2}$. The ${ }^{1,3} \mathrm{D}$ distributions from $\mathrm{Ba}$ metal and $\mathrm{BaF}_{2}$ are broader than the ${ }^{1} S$ groundstate one, indicating that there is interaction of the $6 \mathrm{~s}$ or $5 d$ electron with the substrate. For $\mathrm{BaO}$ the distributions of the $D$ states are similar to the ground state. The calcium process is very similar to the Ba system except that the ${ }^{1,3} D, 4 s^{1} 3 d^{1}$ states of $\mathrm{Ca}$ are not metastable in vacuum and will radiatively decay. In this intermediate case there is partial shielding of, for example, the $3 d^{7}$ electrons in $\mathrm{Fe}\left({ }^{5} F_{J}\right)$ by the $4 s^{1}$ electron.

The first example of the third regime, $\operatorname{In}\left({ }^{2} P_{J}\right)$, is presented here. In this case, the fine-structure states are determined by $\mathbf{L} \cdot \mathbf{S}$ coupling of the outermost electron. The interaction of the $5 p$ electron with the conduction band is strong and the atoms in the ${ }^{2} P_{3 / 2}$ state are effectively deexcited to the ${ }^{2} P_{1 / 2}$ state.

There are implications of this proposed explanation. First, for the well shielded cases, the population of atoms in each state cannot be influenced by the interaction of the departing atom with the surface, but 
rather by some event that occurs in the solid. We propose that the probability of initial excitation of the $d$ electron structure is determined by the amount of the electron-gas disturbance induced by the incoming bombarding particle. ${ }^{17}$ The experimental results of the populations in the high-shielding cases show a dependence on the KE of the incoming ion. ${ }^{3,4}$ Since there is no deexcitation as the atom departs from the surface, as determined from the velocity-distribution shapes, then the measured excited-state populations reflect the excitation process. For the moderate-shielding and exposed cases, the final-state populations reflect both the initial excitation process and the deexcitation as the atoms depart from the surface region.

There are several aspects of these proposed ideas that can be tested and examined further. Obviously, there are other elements and associated fine-structure states that will fit into the three categories. These systems can be investigated. The $a$ priori determination of the interaction strength should be examined. It is not obvious especially at this stage why the ${ }^{1} D$ state of $\mathrm{Ba}$ is partially quenched by the clean metal but not with the oxide. It would be desirable to find other examples like $\operatorname{In}\left({ }^{2} P_{3 / 2}\right)$ where the excited state is completely deexcited.

The authors are grateful to the National Science Foundation, the U.S. Air Force Office of Scientific Research, The U.S. Office of Naval Research, and the IBM Corporation for partial financial support of this work. One of us (B.J.G.) appreciates a TeacherScholar Award from the Camille and Henry Dreyfus Foundation.

(a) Present address: Department of Physics, University of Newcastle, Shortland, N.S.W. 2308, Australia.

(b) Present address: Center for Molecular Electronics,
Carnegie-Mellon University, 4400 Fifth Ave., Pittsburgh, PA 15213.

(c) Present address: Rockwell Science Center, 1049 Camino Dos Rios, Thousand Oaks, CA 91360.

${ }^{1}$ N. A. Yasuf and I. S. T. Tsong, Surf. Sci. 108, 578 (1981).

2W. Husinsky, G. Betz, and I. Girgis, Phys. Rev. Lett. 50, 1689 (1983).

${ }^{3}$ M. J. Pellin, R. B. Wright, and D. M. Gruen, J. Chem. Phys. 74, 6448 (1981).

${ }^{4}$ C. E. Young, W. F. Callaway, M. J. Pellin, and D. M. Gruen, J. Vac. Sci. Technol. A 2, 693 (1984).

${ }^{5}$ M. L. Yu, D. Grischkowsky, and A. C. Balant, Phys. Rev. Lett. 48, 427 (1982).

6B. Schweer and H. L. Bay, Appl. Phys. A 29, 53 (1982).

${ }^{7}$ N. Winograd, J. P. Baxter, and F. M. Kimock, Chem. Phys. Lett. 88, 581 (1982); F. M. Kimock, J. P. Baxter, and N. Winograd, Surf. Sci. 124, L41 (1983); F. M. Kimock, J. P. Baxter, D. L. Pappas, P. H. Kobrin, and N. Winograd, Anal. Chem. 56, 2782 (1984).

8P. H. Kobrin, G. A. Schick, J. P. Baxter, and N. Winograd, Rev. Sci. Instrum. 57, 1354 (1986).

${ }^{9}$ K. P. Huber and G. Herzberg, Constants of Diatomic Molecules (Van Nostrand Reinhold, New York, 1979).

${ }^{10}$ M. A. Douglas, R. M. Hauge, and J. L. Margrave, J. Phys. Chem. 87, 2945 (1983).

11J. P. Baxter, J. Singh, G. A. Schick, P. H. Kobrin, and N. Winograd, Nucl. Instrum. Methods Phys. Res. Sect. B (to be published).

12B. J. Garrison, Nucl. Instrum. Methods Phys. Res. Sect. B (to be published).

${ }^{13}$ F. M. Kimock, Ph.D. dissertation, The Pennsylvania State University, 1984 (unpublished).

${ }^{14}$ N. Winograd, Prog. Solid State Chem. 13, 285 (1982).

${ }^{15}$ E. W. Rothe, V. Krause, and R. Duren, J. Chem. Phys. 72, 5145 (1980); M. J. Janson and S. M. Papernov, J. Phys. B 15, 4175 (1982).

16E. I. Dashevskaya, A. I. Voronin, and E. E. Nikitin, Can. J. Phys. 47, 1237 (1969).

17B. I. Craig and B. J. Garrison, unpublished. 